\section{Allergie gegen Naturlatex: Nur noch ungepudert arbeiten!}

\begin{abstract}
Allergien gegen Naturlatex kommen überwiegend bei Beschäftigten in medizinischen Berufen vor, bedingt durch den Einsatz von mit Maisstärke gepuderten Handschuhen. Neben intensivem Lüften ist der Austausch dieser Handschuhe gegen ungepuderte oder synthetische Handschuhe die effektivste Präventionsmaßnahme.
\end{abstract}

Die Soforttyp-Allergien gegen Naturlatex nehmen deutlich zu. Allerdings begrenzt sich die „Epidemie“ weitgehend auf medizinisches Personal und in geringerem Umfang auf eine kleine Patientengruppe, die Spina-bifida-Kinder. Doch anders als z.B. bei der Neurodermitis kann man bei der Naturlatexallergie den Auslöser gut eingrenzen und effektive Präventionsmaßnahmen benennen, um die Prävalenzzahlen zurückzudrängen.

Untersuchungen haben zweifelsfrei ergeben, dass die Latexallergene aus dem kautschukhaltigen Material der Handschuhe in den heute gebräuchlichen Handschuhpuder übergehen und mit dem Puder beim Auspacken sowie beim Aus- und Anziehen der Handschuhe in die Luft gelangen und so zu inhalativen Allergenen werden. Zwar kann auch ein direkter Hautkontakt mit Latexallergenen Ursache von Latexsensibilisierungen sein, doch ist gerade bei Krankenhausbeschäftigten eine Sensibilisierung über die Atemwege der häufigste Auslöser für Naturlatexallergien.

\section{Maisstärke als Allergenträger}

Eine Querschnittsuntersuchung des Berufsgenossenschaftlichen Forschungsinstituts für Arbeitsmedizin der Ruhruniversität Bochum an Staubproben aus 3 Krankenhäusern und 2 Arztpraxen belegt beispielsweise, dass in der Luft einzelner Räume Latexallergene in klinisch relevanten Mengen (bis zu $714 \mathrm{ng} / \mathrm{m}^{3}$ ) vorkommen. Allergiesymptome der oberen und unteren Atemwege sowie die Bildung von latexspezifischen IgE-Antikörpern war dabei nur bei den Beschäftigten nachzuweisen, die in den Räumen mit

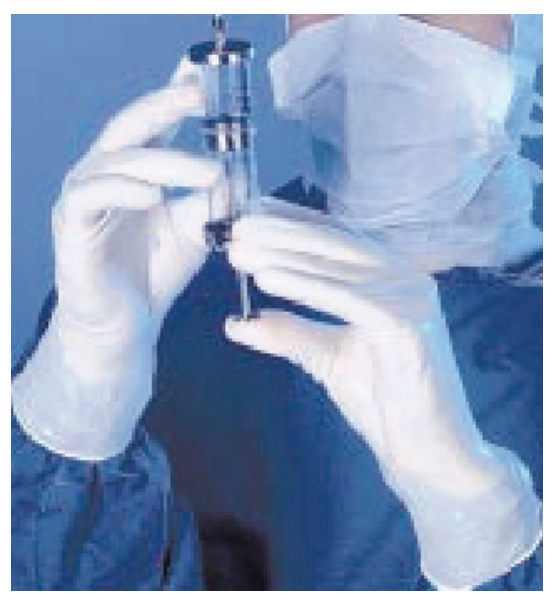

Ungepuderte Naturlatexhandschuheihr Einsatz kann die Luftbelastung mit Latexallergenen in Krankenhäusern und Arztpraxen wirksam reduzieren.

der Latexallergen-belasteten Luft arbeiteten - und praktisch ausschließlich hier wurden die gepuderten und proteinreichen Latexhandschuhe verwendet.

Die Zunahme der Latexsensibilisierungen bei Beschäftigten im Gesundheitswesen ist zum einen mit dem immens gestiegenen Gebrauch von Einmalhandschuhen seit den 80er Jahren zum Schutz vor Infektionen - insbesondere HIV und Hepatitis - zurückzuführen. Ein anderer Grund ist vermutlich auch die Umstellung bei dem verwendeten Handschuhpuder, die zeitgleich erfolgte: Während früher Talkum als Gleitmittel zum Einsatz kam, bediente man sich im Zuge der größeren Nachfrage zunehmend phosphatierter Maisstärke.

Diese modifizierte Stärke dürfte für die Zunahme der Latexsensibilisierungen aus mehreren Gründen verantwortlich sein. Denn ihre Partikel sind im Gegensatz zu denen von Talkum sehr viel leichter und können damit beim An- und Ausziehen der Handschuhe vermehrt in die Luft gelangen. Außerdem sind an Maismehle adsorbierte Allergenproteine im Gegensatz zu den Talkum-adsorbierten Proteinen gut wasserlöslich und haben eine hohe IgE-Bindungsfähigkeit.

\section{Problematische Nassbepuderung}

Über den Weg der Naturlatexallergene aus den Handschuhen in die Maismehle, die unkontaminiert kaum SoforttypSensibilisierungen hervorrufen, wurde bisher nur vereinzelt berichtet. Es ist aber wahrscheinlich, dass das weit verbreitete Nassbepuderungsverfahren für die Übertragung der Latexallergene auf den Handschuhpuder verantwortlich ist (Lundberg M et al. Allergy 50; 1995: 378-80).

Zur Zeit werden verschiedene alternative Herstellungsverfahren getestet, um der Sensibilisierungsproblematik Herr zu werden. So kann trockenes Maismehlpuder direkt auf gut gewaschene Handschuhe aufgetragen oder die Oberfläche mit Polymeren beschichtet werden, wobei man ganz auf die Bepuderung verzichtet.

Mit dem Austausch von allergenreichen gegen allergenarme gepuderte oder ungepuderte latexhaltige oder synthetische Handschuhe ist eine wirkungsvolle Prävention möglich. Studien zeigen, dass innerhalb von nur 24 Stunden nach der Umstellung die Latexallergenkonzentration in der Luft auf Werte unterhalb der Nachweisgrenze fallen.

\section{Ebenfalls hilfreich: Lüften}

Auch ein anderes Verfahren, so die aktuelle Studie des Berufsgenossenschaftlichen Forschungsinstituts, kann die Belastung durch allergenhaltigen Puder beträchtlich reduzieren: Effektives Lüften, z.B. durch ein leistungsfähiges Mehrfiltersystem mit einem Luftdurchsatz von $9000 \mathrm{~m}^{3} /$ Stunde, wie sie in OP-Räumen zum Einsatz kommen.

$b k$

\section{Chen Z, Baur X}

Naturlatexallergengehalt in Gummiartikeln und in der Raumluft von Krankenhäusern und Arztpraxen.

Allergologie 1999; 22: 598-605. 